\title{
Consistently High Unprotected Anal Intercourse (UAI) and factors correlated with UAl among men who have sex with men: implication of a serial cross-sectional study in Guangzhou, China
}

Weibin Cheng ${ }^{1+}$, Weiming Tang ${ }^{2 \dagger}$, Fei Zhong ${ }^{1,3}$, Giridhar R Babu ${ }^{4}$, Zhigang Han ${ }^{1}$, Faju Qin ${ }^{1}$, Kai Gao ${ }^{1}$, Huixia Mai ${ }^{1}$, Yuteng Zhao ${ }^{1}$, Caiyun Liang ${ }^{1}$, Lirui Fan ${ }^{1}$, Hao Wu${ }^{1}$, Huifang $\mathrm{Xu}^{1 *}$ and Ming Wang ${ }^{1}$

\begin{abstract}
Background: China experiencing an increasing HIV epidemic among men who have sex with men (MSM), and unprotected anal intercourse (UAI) has played a key role in this process. The aims of this study were to examine the trend of UAI and to explore the factors correlated with UAI among MSM in Guangzhou, China.

Methods: Data from 2008 to 2013 were retrieved from the annual serological and behavioral surveys system. We collected information on demographic, HIV related sexual behavior with men and women, access to HIV prevention services, and symptoms of sexually transmitted infections. Chi-square test was used to examine the similarity of the participants during the study period. Univariate and multivariate logistic regression were conducted to test the factors associated with UAI. Trend test was used to check the change of UAI in different characteristic stratums during the study period.

Results: In total, $58.4 \%$ (range from $54.5 \%$ to $62.0 \%$ ) of the participants reported that they engaged in UAl in the past six months. Participants who aged less than 20 [Adjusted Odds Ratio (AOR): 2.22, 95\% Confidential Interval (CI): 1.07-4.61], only attended elementary school (or less) (AOR: 1.41, 95\% Cl: 1.04-1.90), cohabiting with male partner (AOR: $2.15,95 \%$ Cl: 1.66-2.79), divorced or widowed (AOR: 2.80, 95\% Cl: 1.54-5.07), did not test for HIV in the past year (AOR: 1.36, 95\% Cl: 1.12-1.65), and had 10 or more partners in the past six months (AOR: 1.85, 95\% Cl: 1.18-2.91) had higher odds of UAI. However, the proportions of UAI were stable in different stratums during the study period.

Conclusions: The proportion of MSM engaged in UAI was consistently high during the study period. Effective intervention strategies, which include but not limit to risk reduction counseling and testing services, are urgently needed to bring down the risk behaviors of the MSM in Guangzhou, in order to control HIV/STIs epidemic in this specific population.
\end{abstract}

Keywords: Unprotected anal intercourse (UAI), Men who have sex with men (MSM), Human immunodeficiency virus (HIV), Trend, Serial cross-sectional study, China

\footnotetext{
*Correspondence: xuhuifang1027@21cn.com

${ }^{\dagger}$ Equal contributors

'Department of AIDS/STD Control and Prevention, Guangzhou Center for Disease Control and Prevention, No.1, Qide Road, Jiahe, Baiyun District,

Guangzhou, Guangdong Province 510440, China

Full list of author information is available at the end of the article
} 


\section{Background}

Generally, the Chinese society ascribes negative connotation to homosexual as aberrant behavior [1]. Men who have sex with men (MSM) continue to lead clandestine risk behaviors while they continue to stay married and have children [2]. Even lots of effort already put, we still confront increasing HIV/AIDS epidemic among MSM $[3,4]$, while other high risk groups have been met with greater success [5]. It is estimated that there are nearly 10 million MSM in China $[3,4,6]$. The raising epidemic of HIV and sexually transmitted infections (STIs) may further worsen the burden of HIV/STIs in China $[7,8]$. Targeting intervention could bring down this burden, and there is also evidence available on different sexual risk behavior among MSM [4]. It is estimated that the proportion of MSM engaging in high-risk sexual behavior is changing over a period of time [9]. However, a clear pattern of these changes is yet to be studied in different geographical areas in China.

It is recognized that MSM have high proportion of risky behaviors such as unprotected sex, drug use, and several adverse health outcomes [10]. The enormity of the HIV epidemic warrants for studies and interventions targeting MSM. Our earlier study indicated that unprotected anal intercourse (UAI) significantly decreased as result of risk reduction counseling [4]. However, it is unclear whether such change in behavior was confounded by other intervention efforts.

Mostly, MSM are treated as a homogenous group and therefore the varied risk propensity based on contextual factors are often ignored [11]. UAI is consider to be the key reason of HIV and other STIs transmission among MSM of China [10]. Therefore, it is important to explore the contextual determinants of UAI, as they will provide tools for detection of potential HIV transmission.

Guangzhou is the capital and largest city of Guangdong province; it is also the home 12.78 million people. A serial cross-sectional study was conducted between 2008 and 2013 in Guangzhou, China. HIV risk-reduction counseling and testing was implemented for each participant during each visit of the participants. This counseling procedure included pre- and post-test counseling services. The objectives of the study were to explore the risk behaviors and determinants of HIV/syphilis acquisition in Guangzhou, and assessed the effectiveness of a counseling session in reducing the risk behaviors of MSM.

\section{Methods}

\section{Materials and methods}

The current reported serial cross-sectional study was conducted between 2008 and 2013 in Guangzhou, China.

\section{Sample size}

In each year of the study period, in order to observe the behaviors of MSM in Guangzhou, China, a certain number of MSM were recruited. The sample size that we needed at each year was estimated based on the following formula [12], by using the known HIV epidemic of Guangzhou:

$$
n=[D E F F * N p(1-p)] /\left[\left(d^{2} / Z^{2}{ }_{1-\alpha / 2} *(N-1)+p *(1-p)\right)\right.
$$

From our previous data (unpublished, using the method of capture-recapture), we estimated that there were about 35,000 MSM in Guangzhou at the year of 2008. By using HIV prevalence of $5.2 \%$ in 2008 [13], confidence limit (d) of $5 \%$, a design effect (DEFF) of 2 , and an alpha $(\alpha)$ of 0.05 , a total of $152 \mathrm{MSM}$ were needed. However, in order to increase the power of our study, we decided to increase the sample size to 400, instead of 152. In 2013, Guangzhou was selected as one of the MSM incidence study sites by National Center for AIDS/STD Control and Prevention, and the program need at least $600 \mathrm{MSM}$, thus we further recruited 200 more participants and included them into our current reported study, in order to further increase the power of our study.

\section{Participant recruitment}

Our study was conducted between April and July of each year between the years of 2008 and 2013. In order to be comfortable and convenient to the participants, our study was conducted within office site of a local gay community (Guangzhou Tongzhi). This site was supported by Guangzhou Center for Disease Control and Prevention (CDC), and it was listed as one HIV voluntary counseling and testing site of Guangzhou city. During the study period, the same interviewers conducted the survey by following the same study protocol which developed by Guangzhou CDC. This site is the main site that targeting MSM in Guangzhou, and it tested about 6000 to 8000 MSM annually, which accounted for about $90 \%$ of the MSM tested in Guangzhou at each year. In order to meet the inclusion criteria, the participants must be male, had anal or oral sex with men in the last 12 months, at least 18 years old and had lived in Guangzhou for at least 6 months before our study. In order to increase the representative of our study, snowball sampling method was used to recruit the eligible participants at each year. To encourage the participation of eligible MSM, incentive (A gift of 2 US dollars and cash about 3 US dollars) was given to each participated MSM.

\section{Behavioral Measures}

Face-to-face interview was used to collect information from participants after the blood drawing. Information 
was collected regarding details on demographic characteristics, knowledge and attitudes about HIV, preventive services, recent sexual behaviors and drug use of the participants. Coverage of HIV intervention was defined as the percentage of participants who received any kind of HIV related service from CDC in the past one year. In our study, UAI was defined as inconsistent use of condoms during anal sex with male partners in the past six months. UVI was defined as inconsistent use of condoms during vaginal sex with female partners in the past six months.

We offered HIV risk-reduction counseling and testing for each participant, which included pre- and post-test counseling services. If the participants were HIV positive, he would be referred to the Chinese HIV/AIDS Care program. If the participants were syphilis positive, they will be referred to the STIs clinics at Guangzhou for syphilis treatment.

The ethics Committee, Guangzhou CDC reviewed and approved the study protocol. All participants were provided written informed consent, and had the right to withdraw from the study at any time.

\section{Serologic measures}

Subsequent to signing the written informed consent, $5 \mathrm{ml}$ intravenous blood was drawn from each participant ahead of the interview. HIV screening was done by using enzyme-linked immunoassays (ELISAs; Diagnostic Kit for Antibody to Human Immunodeficiency Virus, BioMérieux, Boxtel, The Netherlands, Beijing BGI-GBI Biotech, Beijing, China). After screened to be HIV positive by ELISA method, confirmation was done by Western Blot (WB;MP Biomedicals Asia Pacific Pte Ltd, Singapore). Syphilis screening was performed by rapid plasma reagents (RPR; Shanghai Kehua Bioengineering Co. Ltd, Shanghai, China). Specimens tested positive by RPR were confirmed by the Treponema Pallidum particle agglutination test (TPPA; Livzon Group Reagent Factory, Zhuhai, China). In our study, syphilis positive was defined as both RPR and TPPA positive.

\section{Data analysis}

Data was double entered and cleaned by using EpiData (version 3.1, Denmark) and Microsoft excel. Statistical analysis was performed by using IBM SPSS Statistic for windows version 18 (SPSS Inc., Chicago, USA). Descriptive analysis was conducted to determine the distribution of the demographic factors and behaviors of the participants. Chi-square test was used to examine the similarity of the participants during the study period. Univariate and multivariate logistic regression were conducted to test the factors associated with UAI. Stepwise method was used in our multivariate logistic regression. The independent variable in the univariate model included age, marital status, Hukou (registered permanent residence), education level, ethnicity, monthly income, age at first sex with male, sexual orientation, venues for meeting partners, UVI, HIV testing history, and preventive intervention services received. The variables that had P-value less than 0.10 during the preliminary analysis were included in the multivariate model.

In order to examine the change of UAI within different strata of interesting factors, we further stratified the participants based on the characters that correlated with UAI from the logistic regression model. After the stratification, we calculated the percentage of UAI at each stratum in each year, and we further performed trend test to check whether the percentage of UAI was changed in different stratums during the study period. All statistical significance test results are reported as p-values, where less than 0.05 was used to define significance.

\section{Results}

In our study, a total of 2603 participants were recruited between the year of 2008 and 2013. The total number of participant recruited annually gradually increased during the study period, even there was a lit bit of wane (Table 1).

\section{Study population characteristics and behaviors}

Among the participants, more than three fifth were aged less than 30, and these percentages did not vary too much during the study period. About $63 \%$ of the participants reported that they were single. However, this percentage increased during the study period $(54.5 \%$ in $2008,60.3 \%$ in $2009,63.2 \%$ in $2010,65.0 \%$ in 2011 , $64.3 \%$ in 2012 and $67.9 \%$ in $2013, \mathrm{P}<0.01$ ). Our study also indicated that less than one third of the participants were residents of Guangzhou, even this percentage varied year by year (Table 1). About $62 \%$ of the participants received high education, and the proportion of the participants attended college or higher slightly increased during the study period $(\mathrm{P}<0.01)$. In total, about $30 \%$ of the participants had monthly income less than RMB 2000 Yuan (US \$320).

About two thirds of the participants admitted that their sexual orientation was homosexual, which ranged between $61.2 \%$ and $71.2 \%$ during the study period. In addition, about three quarters of the participants mainly found their sexual partners online, which was also varied year by year $(65.2 \%$ in $2008,70.9 \%$ in $2009,73.6 \%$ in 2010, $77.3 \%$ in $2011,70.3 \%$ in 2012 and $89.1 \%$ in 2013 , $\mathrm{P}<0.01$ ). Table one also indicated that about $18 \%$ of the participants reported that they started to engage in sex male before 19 years old.

In our study, about one third of the participants reported that they only had one male partner in the past 
Table 1 Demographic characteristics and key behaviors of MSM in Guangzhou, China, 2008-2013

\begin{tabular}{|c|c|c|c|c|c|c|c|c|c|}
\hline Characteristics & $\begin{array}{l}2008 \\
(\mathrm{~N}=379) \\
\mathrm{n}(\%)\end{array}$ & $\begin{array}{l}2009 \\
(\mathrm{~N}=385) \\
\mathrm{n}(\%)\end{array}$ & $\begin{array}{l}2010 \\
(\mathrm{~N}=405) \\
n(\%)\end{array}$ & $\begin{array}{l}2011 \\
(\mathrm{~N}=400) \\
n(\%)\end{array}$ & $\begin{array}{l}2012 \\
(\mathrm{~N}=401) \\
\mathrm{n}(\%)\end{array}$ & $\begin{array}{l}2013 \\
(N=633) \\
n(\%)\end{array}$ & $\begin{array}{l}\text { Total } \\
(\mathrm{N}=2603) \\
\mathrm{n}(\%)\end{array}$ & $\mathrm{x}^{2}$ value & $p^{a}$ \\
\hline Age (years) & & & & & & & & 22.80 & 0.299 \\
\hline$<20$ & $14(3.7)$ & $10(2.6)$ & $13(3.2)$ & $15(3.8)$ & $9(2.2)$ & $15(2.4)$ & $76(2.9)$ & & \\
\hline $20-29$ & $217(57.3)$ & $227(59.0)$ & $245(60.5)$ & $250(62.5)$ & $228(56.9)$ & $385(60.8)$ & $1552(59.6)$ & & \\
\hline $30-39$ & $111(29.3)$ & $113(29.4)$ & $120(29.6)$ & $101(25.3)$ & $128(31.9)$ & $174(27.5)$ & $747(28.7)$ & & \\
\hline $40-49$ & $35(9.2)$ & $27(7.0)$ & $23(5.7)$ & $25(6.3)$ & $24(6.0)$ & $49(7.7)$ & $183(7.0)$ & & \\
\hline$\geq 50$ & $2(0.5)$ & $8(2.1)$ & $4(1.0)$ & $9(2.3)$ & $12(3.0)$ & $10(1.6)$ & $45(1.7)$ & & \\
\hline Marital status & & & & & & & & 40.73 & $<0.001$ \\
\hline Single & $214(54.5)$ & $232(60.3)$ & $256(63.2)$ & $260(65.0)$ & $258(64.3)$ & $430(67.9)$ & $1650(63.4)$ & & \\
\hline Cohabiting with male partner & $67(17.7)$ & $69(17.9)$ & $62(15.3)$ & $46(11.5)$ & $63(15.7)$ & $104(16.4)$ & $411(15.8)$ & & \\
\hline Married & $79(20.8)$ & $69(17.9)$ & $74(18.3)$ & $89(22.3)$ & $69(17.2)$ & $76(12.0)$ & $456(17.5)$ & & \\
\hline Divorced or widowed & $19(5.0)$ & $15(3.9)$ & $13(3.2)$ & $5(1.3)$ & $11(2.7)$ & $23(3.6)$ & $86(3.3)$ & & \\
\hline Hukou (registered permanent residence) & & & & & & & & 57.46 & $<0.001$ \\
\hline Guangzhou & $105(27.7)$ & $122(31.7)$ & $122(30.1)$ & $115(28.8)$ & $134(33.4)$ & $235(37.1)$ & $833(32.0)$ & & \\
\hline Other city in Guangdong province & $85(22.4)$ & $84(21.8)$ & $90(22.2)$ & $67(16.8)$ & $94(23.4)$ & $186(29.4)$ & $606(23.3)$ & & \\
\hline Outside Guangdong province & $189(49.9)$ & $179(46.5)$ & $193(47.7)$ & $218(54.5)$ & $173(43.1)$ & $212(33.5)$ & $1164(44.7)$ & & \\
\hline Han nationality & $362(95.5)$ & $369(95.8)$ & $382(94.3)$ & $381(95.3)$ & $389(97.0)$ & $614(97.0)$ & $2497(95.9)$ & 6.37 & 0.272 \\
\hline Education level & & & & & & & & 64.16 & $<0.001^{\mathrm{C}}$ \\
\hline Junior high school or lower & $72(19.0)$ & $47(12.2)$ & $66(16.3)$ & $53(13.3)$ & $49(12.2)$ & $35(5.5)$ & $322(12.4)$ & & \\
\hline Senior high school & $111(29.3)$ & $99(25.7)$ & $115(28.4)$ & $140(35.0)$ & $103(25.7)$ & $110(17.4)$ & $678(26.0)$ & & \\
\hline College or higher & $196(51.7)$ & $239(62.1)$ & $224(55.3)$ & $207(51.8)$ & $249(62.1)$ & $488(77.1)$ & 1603(61.6) & & \\
\hline Monthly income (CNY) & & & & & & & & 131.60 & $<0.001^{c}$ \\
\hline 0 & $49(12.9)$ & $54(14.0)$ & $46(11.4)$ & $39(9.8)$ & $56(14.0)$ & $84(13.3)$ & $328(12.6)$ & & \\
\hline$\leq 2000$ & $125(33.0)$ & $88(22.8)$ & $99(24.5)$ & $65(16.3)$ & $27(6.7)$ & $47(7.4)$ & $451(17.3)$ & & \\
\hline $2001-3000$ & $85(22.4)$ & $95(24.7)$ & $94(23.2)$ & $112(28.0)$ & $78(19.5)$ & $98(15.5)$ & $562(21.6)$ & & \\
\hline $3001-4000$ & $60(15.8)$ & $60(15.6)$ & $69(17.0)$ & $86(21.5)$ & $72(18.0)$ & $112(17.7)$ & $459(17.6)$ & & \\
\hline$>4000$ & $60(15.8)$ & $88(22.9)$ & $97(24.0)$ & $98(24.5)$ & $168(41.9)$ & $292(46.1)$ & $803(30.8)$ & & \\
\hline Self-perceived sexual orientation & & & & & & & & 26.23 & 0.003 \\
\hline Homosexual & $232(61.2)$ & $247(64.2)$ & $264(65.2)$ & $260(65.0)$ & $247(61.6)$ & $451(71.2)$ & $1701(65.3)$ & & \\
\hline Bisexual & $106(28.0)$ & $109(28.3)$ & $117(28.9)$ & $120(30.0)$ & $119(29.7)$ & $148(23.4)$ & $719(27.6)$ & & \\
\hline Uncertain & $41(10.8)$ & $29(7.5)$ & $24(5.9)$ & $20(5.0)$ & $35(8.7)$ & $34(5.4)$ & $183(7.0)$ & & \\
\hline Venues for meeting partners & & & & & & & & 170.40 & $<0.001$ \\
\hline Bar, disco, tea house or club & $28(7.4)$ & $31(8.1)$ & $25(6.2)$ & $27(6.8)$ & $22(5.5)$ & $22(3.5)$ & $155(6.0)$ & & \\
\hline Bath house, sauna or massage & $25(6.6)$ & $15(3.9)$ & $14(3.5)$ & $9(2.3)$ & $6(1.5)$ & $8(1.3)$ & $77(3.0)$ & & \\
\hline Park, public toilet & $57(15.0)$ & $25(6.5)$ & $24(5.9)$ & $18(4.5)$ & $40(10.0)$ & $5(0.8)$ & $169(6.5)$ & & \\
\hline Internet & $247(65.2)$ & $273(70.9)$ & $298(73.6)$ & $309(77.3)$ & $282(70.3)$ & $564(89.1)$ & $1973(75.8)$ & & \\
\hline Other & $22(5.8)$ & $41(10.6)$ & $44(10.9)$ & $37(9.3)$ & $51(12.7)$ & $34(5.4)$ & $229(8.8)$ & & \\
\hline Age of first sex with male (years) & & & & & & & & 1.97 & $0.161^{c}$ \\
\hline$<19$ & $66(17.4)$ & 88 (22.9) & 63 (15.6) & $62(15.5)$ & 70 (17.5) & 118 (18.6) & 467 (17.9) & & \\
\hline $19-24$ & $195(51.5)$ & $178(46.2)$ & $181(44.7)$ & $179(44.8)$ & $192(48.0)$ & $364(57.5)$ & 1289 (49.5) & & \\
\hline$\geq 25$ & 118 (31.1) & 119 (30.9) & 161 (39.8) & 159 (39.8) & $138(34.5)$ & $151(23.9)$ & 846 (32.5) & & \\
\hline
\end{tabular}




\begin{tabular}{|c|c|c|c|c|c|c|c|c|c|}
\hline \multicolumn{8}{|l|}{ Number of male partners in the past 6 months } & \multirow[t]{6}{*}{7.45} & \multirow[t]{6}{*}{$0.006^{c}$} \\
\hline 1 & $94(28.0)$ & $123(35.3)$ & $121(33.6)$ & $123(33.3)$ & $136(37.5)$ & $184(32.2)$ & $781(33.3)$ & & \\
\hline 2 & $80(23.8)$ & $92(26.4)$ & $96(26.7)$ & $98(26.6)$ & $97(26.7)$ & $159(27.8)$ & $622(26.5)$ & & \\
\hline 3 & $69(20.5)$ & $66(19.0)$ & $63(17.5)$ & $66(17.9)$ & $72(19.8)$ & $112(19.6)$ & $448(19.1)$ & & \\
\hline $4-9$ & $69(20.5)$ & $38(10.9)$ & $60(16.7)$ & $68(18.4)$ & $40(11.1)$ & $105(18.4)$ & $380(16.2)$ & & \\
\hline$\geq 10$ & $24(7.1)$ & $29(8.3)$ & $20(5.6)$ & $14(3.8)$ & $18(5.0)$ & $12(2.1)$ & $117(5.0)$ & & \\
\hline Had sex with female in the past six months & $82(21.6)$ & $81(21.0)$ & $80(19.8)$ & $74(18.5)$ & $87(21.7)$ & $76(12.0)$ & $480(18.4)$ & 25.02 & $<0.001$ \\
\hline$u V l^{b}$ & $58(15.3)$ & $49(12.7)$ & $64(15.8)$ & $52(13.0)$ & $60(15.0)$ & $53(8.4)$ & $336(12.9)$ & 18.05 & 0.003 \\
\hline$U A I^{b}$ & $233(61.5)$ & $210(54.5)$ & $240(59.3)$ & $248(62.0)$ & $227(56.6)$ & $361(57.0)$ & $1519(58.4)$ & 7.10 & 0.213 \\
\hline HIV test in the past 12 months & $62(16.4)$ & $274(71.2)$ & $178(44.0)$ & $177(44.7)$ & $198(49.7)$ & $338(53.4)$ & $1227(47.3)$ & 247.15 & $<0.001$ \\
\hline Coverage of HIV intervention & $287(75.7)$ & $338(87.8)$ & $301(74.3)$ & $283(70.8)$ & $334(83.3)$ & $538(85.0)$ & $2081(79.9)$ & 60.94 & $<0.001$ \\
\hline
\end{tabular}

${ }^{\text {apearson }}$ chi-square test.

bUAI, unprotected anal intercourse; UVI, unprotected vaginal intercourse.

'Linear-by-linear association chi-square test.

six months. This proportion also varied year by year (ranged between 28.0\% in 2008 and 37.5 in 2012, $\mathrm{P}=0.01$ ). In addition, about $13 \%$ and $58 \%$ of the participants reported that they engaged in UVI and UAI in the past six months, respectively. About 47\% (ranged between 16.4\% and $71.2 \%, \mathrm{P}<0.01$ ) of the participants tested for HIV in the past 12 months. In addition, about four fifth of the participants reported that they received HIV related intervention.

\section{Factors correlated with UAI}

In our study, both univariate and multivariate model shown that compared with those participants who aged 40 or more, the participants aged less than 20 had significant higher odds of engaging in UAI (Crude Odds Ratio $(\mathrm{COR})=2.75$, 95\% CI:1.55-4.88, Adjusted Odds Ratio $(\mathrm{AOR})=2.22,95 \% \mathrm{CI}: 1.07-4.61)$. And the univariate model indicated that the risk of engaging in UAI increased with the decreasing of age (Table 2). Same phenomenon was also observed for education, with poorer education status of the participants associated with the higher odds of engaging in UAI. The adjusted model showed that the odds of the participants who only attended elementary school (or less) was about 1.41 (95\% CI: 1.04-1.90) times of the odds of those attended college.

The crude and adjusted model also indicated that compared with those who were single, the participants who were cohabiting with male partner, divorced or widowed had significantly higher odds of engaging in UAI, with AORs of 2.15 (95\% CI: 1.66-2.79) and 2.80 (95\% CI: 1.54-5.07), respectively. Table 2 also pointed out that those who did not test for HIV in the past year $(\mathrm{AOR}=1.36,95 \% \mathrm{CI}: 1.12-1.65)$ or did not receive any HIV related services (AOR $=1.21,95 \%$ CI: 0.94-1.56) had higher odds of engaging in UAI, despite the latter association being not significant in the adjusted model. In addition, the risk of having UAI increased with the increasing number of male partners in the past six months. For example, the odds of engaging in UAI was $85 \%$ higher for those who had 10 or more partners in the past six months (OR: 1.85, 95\% CI: 1.18-2.91) compared to those who had only one partner in the past six months.

The results of our study indicated that the age of first sex with male did not correlate with UAI. The stratified trend analysis (Table 3) demonstrated that the proportions of UAI in different stratums were stable during the study period, and the P-value were greater than 0.05 . For example, UAI was stable among the participants who were aged between 20-29, single, and attended college or above, barring slight fluctuations.

\section{Discussion}

In this serial cross-sectional study conducted in Guangzhou, China, we found a high proportion of MSM engaged in UAI in each year, and this proportion did not change significantly in the past six years. We further found that younger age, lower education, cohabiting with male partner, widowed or divorced, not tested for HIV in the last year, and having more than one male partner in the past six months were associated with UAI. These findings were very important for the design of targeting intervention strategies, in order to bring down the high rate of UAI, and in turn, reduce the chance of HIV and other STIs transmission.

Our study indicated that the overall UAI rate was high in Guangzhou, and this result is almost the same as one study conducted by our team in 2008 [13]. Similar results were also reported in several other cities in China. For example, Tang W et al. reported that the UAI rate was $62.3 \%$ in Nanjing at the year of 2008 [14]. Feng L 
Table 2 Factors associated with unprotected anal intercourse among MSM in Guangzhou, 2008-2013, by Logistic regression model $^{\mathrm{a}}$

\begin{tabular}{|c|c|c|c|}
\hline Factor & $\mathrm{UAI}^{\mathrm{b}} \%(\mathrm{n})$ & $\operatorname{COR}(95 \% \mathrm{Cl})^{\mathrm{c}}$ & AOR $(95 \% \mathrm{Cl})^{\mathrm{d}}$ \\
\hline \multicolumn{4}{|l|}{ Age (years) } \\
\hline$<20$ & $73.7(56)$ & $2.75(1.55-4.88)^{f}$ & $2.22(1.07-4.61)^{e}$ \\
\hline 20-29 & $59.0(916)$ & $1.42(1.07-1.87)^{\mathrm{e}}$ & $1.30(0.87-1.93)$ \\
\hline $30-39$ & $57.8(432)$ & $1.35(1.00-1.81)$ & $1.17(0.81-1.67)$ \\
\hline$\geq 40$ & $50.4(115)$ & 1.00 & 1.00 \\
\hline \multicolumn{4}{|l|}{ Ethnicity } \\
\hline Han & $58.5(1461)$ & 1 & - \\
\hline Others & $54.7(58)$ & $0.86(0.58-1.27)$ & \\
\hline \multicolumn{4}{|l|}{ Hukou (registered permanent residence) } \\
\hline Guangzhou & $56.7(472)$ & 1 & - \\
\hline Other city in Guangdong province & $58.4(354)$ & $1.07(0.87-1.33)$ & \\
\hline Outside Guangdong province & $59.5(693)$ & $1.13(0.94-1.35)$ & \\
\hline \multicolumn{4}{|l|}{ Education level } \\
\hline Junior high school or lower & $63.4(204)$ & $1.35(1.06-1.73)^{\mathrm{e}}$ & $1.41(1.04-1.90)^{\mathrm{e}}$ \\
\hline Senior high school & $61.2(415)$ & $1.23(1.03-1.48)^{\mathrm{e}}$ & $1.23(0.99-1.51)$ \\
\hline College or higher & $56.1(900)$ & 1.00 & 1.00 \\
\hline \multicolumn{4}{|l|}{ Marital status } \\
\hline Single & $54.7(903)$ & 1.00 & 1.00 \\
\hline Cohabiting with male partner & $72.0(296)$ & $2.13(1.68-2.70)^{f}$ & $2.15(1.66-2.79)^{f}$ \\
\hline Married & $56.8(259)$ & $1.08(0.88-1.34)$ & $1.28(0.96-1.71)$ \\
\hline Divorced or widowed & $70.9(61)$ & $2.02(1.26-3.25)^{f}$ & $2.80(1.54-5.07)^{f}$ \\
\hline \multicolumn{4}{|l|}{ Monthly income (CNY) } \\
\hline 0 & $60.4(198)$ & 1 & - \\
\hline$\leq 2000$ & $62.5(282)$ & $1.10(0.82-1.47)$ & \\
\hline $2001-3000$ & $57.3(322)$ & $0.88(0.67-1.16)$ & \\
\hline $3001-4000$ & $57.3(263)$ & $0.88(0.66-1.16)$ & \\
\hline$>4000$ & $56.5(454)$ & $0.85(0.66-1.11)$ & \\
\hline \multicolumn{4}{|l|}{ Self-perceived sexual orientation } \\
\hline Homosexual & $59.5(1012)$ & 1 & - \\
\hline Bisexual & $57.0(470)$ & $0.90(0.76-1.08)$ & \\
\hline Uncertain & $53.0(97)$ & $0.77(0.57-1.04)$ & \\
\hline \multicolumn{4}{|l|}{ Venues for meeting partners } \\
\hline Bar, disco, tea house or club & $60.6(94)$ & 1 & - \\
\hline Bath house, sauna or massage & $61.0(47)$ & $1.02(.058-1.780$ & \\
\hline Park, public toilet & $55.4(96)$ & $0.81(0.52-1.27)$ & \\
\hline Internet & $58.5(1155)$ & $0.92(0.66-1.28)$ & \\
\hline Other & $56.3(129)$ & $0.84(0.55-1.27)$ & \\
\hline \multicolumn{4}{|l|}{ Age of first sex with male (years) } \\
\hline$<19$ & $63.8(298)$ & 1.00 & 1.00 \\
\hline $19-24$ & $58.7(756)$ & $0.80(0.65-1.00)$ & $0.93(0.73-1.20)$ \\
\hline$\geq 25$ & $55.0(465)$ & $0.69(0.55-0.87)^{f}$ & $0.78(0.58-1.05)$ \\
\hline
\end{tabular}




\begin{tabular}{|c|c|c|c|}
\hline \multicolumn{4}{|c|}{ HIV test in the past 12 months } \\
\hline Yes & $55.3(679)$ & 1.00 & 1.00 \\
\hline no & $61.1(834)$ & $1.28(1.08-1.48)^{f}$ & $1.36(1.12-1.65)^{f}$ \\
\hline \multicolumn{4}{|l|}{$U V^{b}$} \\
\hline Yes & $61.9(208)$ & $1.16(0.94-1.50)$ & \\
\hline No & $57.8(1311)$ & 1 & - \\
\hline \multicolumn{4}{|c|}{ Coverage of HIV intervention } \\
\hline Yes & $57.0(1186)$ & 1.00 & 1.00 \\
\hline no & $63.8(333)$ & $1.33(1.09-1.62)^{f}$ & $1.21(0.94-1.56)$ \\
\hline \multicolumn{4}{|c|}{ Number of male partners in the past 6 months } \\
\hline 1 & $59.3(463)$ & 1.00 & 1.00 \\
\hline 2 & $63.3(394)$ & $1.18(0.96-1.47)$ & $1.27(1.02-1.59)^{\mathrm{e}}$ \\
\hline 3 & $67.4(302)$ & $1.42(1.11-1.81)^{f}$ & $1.49(1.16-1.92)^{f}$ \\
\hline $4-9$ & $71.6(272)$ & $1.73(1.33-2.25)^{f}$ & $1.77(1.35-2.33)^{f}$ \\
\hline$\geq 10$ & $73.5(86)$ & $1.91(1.23-2.94)^{f}$ & $1.85(1.18-2.91)^{f}$ \\
\hline
\end{tabular}

${ }^{a}$ Only factors significant in univariate analysis were included in the multivariate logistic regression model.

bunprotected anal intercourse.

'Univariate logistic regression analysis; ${ }^{\mathrm{d}}$ Multivariate logistic regression analysis.

${ }^{\mathrm{e}} P<0.05 ;{ }^{\mathrm{f}} P<0.01$.

reported that the UAI rate was about $62 \%$ in Chongqing in 2010 [15]. Recently, an updated meta-analysis summarized that the overall UAI rate among Chinese MSM was about $53 \%$ (95\% CI: 51-56\%) [16], which is lower than the overall proportion of UAI in Guangzhou.

Besides this, our study also found that the UAI proportion was consistently high during the study period, which indicated that the intervention implemented in the past few years did not bring down the high-risk behaviors of the MSM in Guangzhou. This may explain why the HIV prevalence consistently increased in Guangzhou during 2008 and 2013 (Increased from 5.2\% in 2008 [13] to $11.4 \%$ in 2013 [8]). Thus, an effective intervention method that can change the behaviors of MSM is urgently needed since UAI is a well-known risk factor of HIV and other STIs [17-19].

Given the high HIV prevalence and incidence (about 10/100 person-years, unpublished data) among MSM in Guangzhou, the consistent high UAI rate is an important signal to us. This consistent high risk behavior may further fast the spreading of HIV transmission among MSM in Guangzhou. Effective intervention programs targeting this high risk behavior are urgently needed. Risk-reductioncounseling, which is considered to be an effective intervention method, that could bring down the risk behaviors of MSM should be adapted and used. In addition, other intervention methods include but not limited to condom promotion and distribution, internet-facilitated interactive intervention (developed by our study team, unpublished data) and HIV screening also should be implemented.
The results of our study also indicated that $18 \%$ of the participants were married, and about $13 \%$ of the participants engaged in UVI in the past six month. Given the higher HIV prevalence in MSM in Guangzhou (11.4\% in 2013), HIV prevention strategies that prevent the HIV transmission from MSM to their female partners are urgently needed.

Our study found that not being tested for HIV in the past year was associated with UAI. This finding is similar with the result of one study conducted in Scotland [20]. However, this is first study according to our knowledge that reported the correlation between UAI and HIV testing behaviors in China. In addition, lack of HIV related intervention was also positive correlated with UAI. These results indicated that the increasing HIV testing and related intervention could potentially bring down the proportion of UAI in MSM in Guangzhou. In the meantime, HIV testing not only increased the detection ratio of HIV infection, but also provided an opportunity for implementing positive intervention techniques such as serosorting and strategic positioning, which can further reduce the second transmission.

Our study found that younger age, particular for those who aged less than 20, were associated with UAI. Similar result was reported by in San Francisco [21]. One potential reason is that young people may lack of the knowledge of self-protection, and more likely to take risks in the pursuit of excitement. Similar to the findings of a study conducted in Jinan [22], our results too indicated that participants who had multiple sexual partners in the 
Table 3 Characteristics correlated with unprotected anal intercourse among MSM in Guangzhou: trend from 2008 to $2013^{a}$

\begin{tabular}{|c|c|c|c|c|c|c|c|c|}
\hline \multirow[t]{3}{*}{ Characteristics } & \multicolumn{8}{|c|}{ Percentage of UAI in different stratums } \\
\hline & 2008 & 2009 & 2010 & 2011 & 2012 & 2013 & \multirow[t]{2}{*}{$x^{2}$ value } & \multirow[t]{2}{*}{$P^{b}$} \\
\hline & $\%(n)$ & $\%(n)$ & $\%(n)$ & $\%(n)$ & $\%(n)$ & $\%(n)$ & & \\
\hline \multicolumn{9}{|l|}{ Age (years) } \\
\hline$<20$ & $64.3(9)$ & $70.0(7)$ & $69.2(9)$ & $80.0(12)$ & $88.9(8)$ & $73.3(1)$ & 0.94 & 0.333 \\
\hline $20-29$ & $60.8(132)$ & $55.1(125)$ & $61.2(150)$ & $62.4(156)$ & $55.7(127)$ & $58.7(226)$ & 0.07 & 0.786 \\
\hline $30-39$ & $64.0(71)$ & $54.9(62)$ & $58.3(70)$ & $67.3(68)$ & $55.5(71)$ & $51.7(90)$ & 2.61 & 0.106 \\
\hline$\geq 40$ & $56.8(21)$ & $45.7(16)$ & $40.7(11)$ & $35.3(12)$ & $58.3(21)$ & $57.6(34)$ & 0.55 & 0.458 \\
\hline \multicolumn{9}{|l|}{ Marital status } \\
\hline Single & $58.4(125)$ & $47.8(111)$ & $57.0(146)$ & $60.8(158)$ & $53.5(138)$ & $52.3(225)$ & 0.37 & 0.544 \\
\hline Cohabiting with male partner & $73.1(49)$ & $69.6(48)$ & $64.5(40)$ & $76.1(35)$ & $68.3(43)$ & $77.9(81)$ & 0.88 & 0.349 \\
\hline Married & $58.2(46)$ & $56.5(39)$ & $63.5(47)$ & $56.2(50)$ & $53.6(37)$ & $52.6(40)$ & 0.84 & 0.360 \\
\hline Divorced or widowed & $68.4(13)$ & $80.0(12)$ & $53.8(7)$ & $100.0(5)$ & $81.8(9)$ & $65.2(15)$ & 0.01 & 0.952 \\
\hline \multicolumn{9}{|l|}{ Education level } \\
\hline Junior high school or lower & $59.7(43)$ & $66.0(31)$ & $62.1(41)$ & $62.3(33)$ & $61.2(30)$ & $74.3(26)$ & 0.80 & 0.372 \\
\hline Senior high school & $62.2(69)$ & $55.6(55)$ & $64.3(74)$ & $65.0(91)$ & $59.2(61)$ & $59.1(65)$ & 0.01 & 0.914 \\
\hline College or higher & $61.7(121)$ & $51.9(124)$ & $55.8(125)$ & $59.9(124)$ & $54.6(136)$ & $55.3(270)$ & 0.44 & 0.509 \\
\hline \multicolumn{9}{|c|}{ Number of male partners in the past 6 months } \\
\hline 1 & $69.1(65)$ & $58.5(72)$ & $57.0(69)$ & $58.5(72)$ & $55.1(75)$ & $59.8(110)$ & 1.31 & 0.252 \\
\hline 2 & $60.0(48)$ & $58.7(54)$ & $59.4(57)$ & $70.4(69)$ & $68.0(66)$ & $62.9(100)$ & 1.25 & 0.263 \\
\hline 3 & $69.6(48)$ & $65.2(43)$ & $77.8(49)$ & $72.7(48)$ & $66.7(48)$ & $58.9(66)$ & 2.39 & 0.122 \\
\hline $4-9$ & $75.4(52)$ & $60.5(23)$ & $80.0(48)$ & $70.6(48)$ & $62.5(25)$ & $72.4(76)$ & 0.16 & 0.689 \\
\hline$\geq 10$ & $83.3(20)$ & $62.1(18)$ & $85.0(17)$ & $78.6(11)$ & $72.2(13)$ & $58.3(7)$ & 0.07 & 0.403 \\
\hline \multicolumn{9}{|l|}{ HIV test in the past 12 months } \\
\hline Yes & $64.5(40)$ & $53.3(146)$ & $55.1(98)$ & $55.4(98)$ & $59.1(117)$ & $53.3(180)$ & 0.17 & 0.679 \\
\hline No & 60.9 (193 & $57.7(64)$ & $62.6(142)$ & $67.1(147)$ & $54.3(107)$ & $61.4(181)$ & 0.03 & 0.863 \\
\hline
\end{tabular}

anly significant factors in multivariate logistic regression were analyzed.

${ }^{b}$ Linear-by-linear association chi-square test.

past six month also had higher odds of engaging in UAI. Thus, it is important to consider this evidence in the design of future intervention programs by specifically target in reducing the number of partners.

The results of our study pointed out that cohabiting with male partner was associated with UAI. One possible explanation for this phenomenon is that the cohabited MSM are more likely to have stable or regular partners, and they already established mutual trust with each other, and more likely not to use condoms. Similar results were found for divorced or widowed participants. In addition, our results corroborated earlier studies indicating that poor education is significantly associated with UAI [22]. One possible explanation for this situation is that the participants who received less education may have a poor understanding of health and disease, and therefore are generally do not take precautionary measures [23].
In our study, we observed some interesting findings, for example, less than one third were residents of Guangzhou, approximately one third did not identify as homosexual and the association with increased partner numbers and UAI. These indicated that the proportion of participants who had casual partners may high in Guangzhou. Given the high UAI rate and high HIV prevalence among MSM in Guangzhou, this may spread the transmission of HIV in the future. We should take these phenomena into consideration in our future study design and intervention effort.

The strength of our study was the use of serial crosssectional study design, larger sample size and the longer observational time (six years). As an observational study, our study has certain limitations. First, we did not collect the information on the UAI with different partnerships, thus prevented exploration the factors under different relationship. We also did not differentiate 
unprotected safer sex practices such as serosorting, strategic positioning and withdrawal before ejaculation from UAI, which may limited our ability to understand the current drivers of HIV transmission in the community. Second, despite of using same sampling method during the study period, we still found that the participants were marginally incomparable. For example, some demographic characteristics of the participants were waned year by year. Third, since this is a cross-sectional study design, lacked of temporality prevents us from drawing any causal inferences. Fourth, all of the information collected in our study was based on the self-report, and possible information bias may exist in our study, due to the problem of social desirability. Fifth, we did not collect information on the proportion of the participants who attended the study for more than once, which limited our ability to assess the possible effect of intervention program that we implemented in our study. Moreover, selection bias can also be a potential threat to validity of current reported study, since we did not collect information on the response rate of the eligible participants.

\section{Conclusion}

In conclusion, we found that the proportion of MSM engaged in UAI was consistently high during every year of the study period. Also, younger age, poor education, cohabiting with male partner, and widowed or divorced participants had higher odds of engaging in UAI. More importantly, not being tested for HIV in the past year and having multiple male partners in the past six months were significantly correlated with UAI. Effective intervention strategies are urgently needed to bring down the risk behaviors of the MSM in Guangzhou, in order to control HIV/STIs epidemic in this specific population.

\section{Competing interests}

The authors declare that they have no competing interests.

\section{Authors' contributions}

WC developed study protocol, collected data, analyzed data, interpreted findings and revised the manuscript. WT interpreted the findings and drafted of the manuscript. FZ developed study protocol, collected data, interpreted the findings. BG contributed to draft the manuscript and language check. $\mathrm{ZH}$ prepared the study proposal and supervised data collection. FQ, KG, HM YZ, CL, LF, HW prepared the study proposal, collected data and performed data management. HX supervised the study and provided technical inputs to the manuscript. MW supervised the study and substantially reviewed the manuscript. All authors critically reviewed and approved the final version of this paper for publication.

\section{Acknowledgments}

The authors are indebted to the colleagues from the Department of AIDS/ STD Control and Prevention, Guangzhou Center for Disease Control and Prevention (Wen Fang, Liu Jiewei, Xie Muqun and Tang Chen), and staffs form Lingnan Fellow Community Health Support Center (Liu Qi, Meng Gang, Li Jingyan, Lu Yongheng and Xue Kunyu) for their contribution in helping the community mobilization and implementing of data collection. We also feel grateful for MSM participants for their voluntary participation. The funding of this study was supported by Medical Scientific Research Foundation of Guangdong Province (A2013539), Medical Scientific Program of Guangzhou (20131A011114, 2013A011090012, 20141A011061), and Science and Technology Program of Guangzhou (2012Y2-00021). The funders had no role in the study design, data collection, data analysis, data interpretation, or writing of the manuscript.

\section{Author details}

'Department of AIDS/STD Control and Prevention, Guangzhou Center for Disease Control and Prevention, No.1, Qide Road, Jiahe, Baiyun District, Guangzhou, Guangdong Province 510440, China. ${ }^{2}$ University of North Carolina Project-China, No.2 Lujing Road, Yuexiu District, Guangzhou, Guangdong Province 510095, China. ${ }^{3}$ School of Public Health, Sun Yat-Sen University, No.74 2nd Yat-Sen Road, Yuexiu District, Guangzhou, Guangdong, Province 510080, China. ${ }^{4}$ Department of Epidemiology, Public Health Foundation of India, IIPH H, Bangalore Campus, SIHFW Premises, 1st cross, Magadi road, Bangalore, Karnataka 560023, India.

Received: 22 July 2014 Accepted: 11 December 2014

Published: 18 December 2014

\section{References}

1. Li YH: Subculture of homosexuality. In China Today Press. Beijing China: China Today Press; 1998

2. Zhou YR: Homosexuality, seropositivity, and family obligations: Perspectives of HIV-infected men who have sex with men in China. Cult Health Sex 2006, 8(6):487-500

3. Chen X-S, Peeling RW, Yin Y-P, Mabey DC: The epidemic of sexually transmitted infections in China: implications for control and future perspectives. BMC Med 2011, 9(1):111.

4. Huan X, Tang W, Babu GR, Li J, Zhang M, Liu X, Yan H, Fu G, Zhao J, Yang $\mathrm{H}$ : HIV risk-reduction counseling and testing on behavior change of MSM. PLoS One 2013, 8(7):e69740.

5. Wang L, Tang W, Wang L, Qian S, Li Y-g, Xing J, Li D, Ding Z, Babu GR, Wang N: The HIV, Syphilis, and HCV Epidemics Among Female Sex Workers in China: Results From a Serial Cross-Sectional Study Between 2008 and 2012. Clin Infect Dis 2014: ciu245. [Epub ahead of print].

6. Wong FY, Huang ZJ, Wang W, He N, Marzzurco J, Frangos S, Buchholz ME, Young D, Smith BD: STIs and HIV among men having sex with men in China: a ticking time bomb? AIDS Educ Prev 2009, 21(5):430-446.

7. Zhang L, Chow EP, Jing J, Zhuang X, Li X, He M, Sun H, Li X, Gorgens M, Wilson D: HIV prevalence in China: integration of surveillance data and a systematic review. Lancet Infect Dis 2013, 13(11):955-963.

8. Zhong F, Liang B, Xu H, Cheng W, Fan L, Han Z, Liang C, Gao K, Mai H, Qin F: Increasing HIV and Decreasing Syphilis Prevalence in a Context of Persistently High Unprotected Anal Intercourse, Six Consecutive Annual Surveys among Men Who Have Sex with Men in Guangzhou, China, 2008 to 2013. PLoS One 2014, 9(7):e103136.

9. He Q, Peng WJ, Zhang JQ, Wang BX, Wang J: Prevalence of unprotected anal intercourse and unprotected vaginal intercourse among HIV-positive men who have sex with men in China: a meta-analysis. Sex Transm Infect 2012, 88(3):229-233.

10. Beyrer C, Baral SD, van Griensven F, Goodreau SM, Chariyalertsak S, Wirtz AL, Brookmeyer R: Global epidemiology of HIV infection in men who have sex with men. Lancet 2012, 380(9839):367-377.

11. Jones RH: Imagined comrades and imaginary protections: Identity, community and sexual risk among men who have sex with men in China. J Homosex 2007, 53(3):83-115.

12. Tang W, Yang H, Mahapatra T, Huan X, Yan H, Li J, Fu G, Zhao J, Detels R: Feasibility of recruiting a diverse sample of men who have sex with men: observation from Nanjing, China. PLoS one 2013, 8(11):e77645.

13. Zhong $F$, Lin $P$, Xu H, Wang Y, Wang M, He Q, Fan L, Li Y, Wen F, Liang Y: Possible increase in HIV and syphilis prevalence among men who have sex with men in Guangzhou, China: results from a respondent-driven sampling survey. AIDS Behav 2011, 15(5):1058-1066.

14. Tang W, Huan X, Mahapatra T, Tang S, Li J, Yan H, Fu G, Yang H, Zhao J, Detels $R$ : Factors associated with unprotected anal intercourse among men who have sex with men: results from a respondent driven sampling survey in Nanjing, China, 2008. AIDS Behav 2013, 17(4):1415-1422.

15. Feng L, Ding $X, X u J$, , Ou Y, Xu S, Zheng J, Guo X, Yang M, Liu X: Study on HIV, syphilis and HCV prevalence and its associated factors among 
internet MSM comparison to non-internet MSM in Chongqing. J Trop Med 2010, 10(1):78-82.

16. Wu J, Hu Y, Jia Y, Su Y, Cui H, Liu H, Wang N: Prevalence of Unprotected Anal Intercourse among Men Who Have Sex with Men in China: An Updated Meta-Analysis. PLoS One 2014, 9(5):e98366.

17. Tang W, Yan H, Liu X, Wang N, Zhang M, Zhao X, Zhang J, Li L, Huan X, Yang H: Factors associated with HIV infection among men who have sex with men in Nanjing, Suzhou and Yangzhou: a 1: 4 matched casecontrol study. Zhonghua Liu Xing Bing Xue Za Zhi 2009, 30(5):448-451.

18. Feng Y, Wu Z, Detels R, Qin G, Liu L, Wang X, Wang J, Zhang L: HIV/STD prevalence among MSM in Chengdu, China and associated risk factors for HIV infection. J Acquir Immune Defic Syndr 2010, 53(Suppl 1):S74-S80.

19. Suarez T, Miller J: Negotiating risks in context: a perspective on unprotected anal intercourse and barebacking among men who have sex with men-where do we go from here? Arch Sex Behav 2001, 30(3):287-300.

20. Hart G, Williamson L, Flowers P, Frankis J, Der G: Gay men's HIV testing behaviour in Scotland. AIDS Care 2002, 14(5):665-674.

21. Waldo CR, McFarland W, Katz MH, MacKellar D, Valleroy LA: Very young gay and bisexual men are at risk for HIV infection: the San Francisco Bay Area Young Men's Survey II. J Acquir Immune Defic Syndr 2000, 24(2):168-174

22. Li X, Shi W, Li D, Ruan Y, Jia Y, Vermund SH, Zhang X, Wang C, Liu Y, Yu M: Predictors of unprotected sex among men who have sex with men in Beijing, China. Southeast Asian J Trop Med Public Health 2008, 39(1):99-108.

23. Li H, Lau JT, Holroyd E, Yi H: Sociocultural facilitators and barriers to condom use during anal sex among men who have sex with men in Guangzhou, China: an ethnographic study. AIDS Care 2010, 22(12):1481-1486.

doi:10.1186/s12879-014-0696-8

Cite this article as: Cheng et al:: Consistently High Unprotected Anal Intercourse (UAI) and factors correlated with UAI among men who have sex with men: implication of a serial cross-sectional study in Guangzhou, China. BMC Infectious Diseases 2014 14:696.

\section{Submit your next manuscript to BioMed Central and take full advantage of:}

- Convenient online submission

- Thorough peer review

- No space constraints or color figure charges

- Immediate publication on acceptance

- Inclusion in PubMed, CAS, Scopus and Google Scholar

- Research which is freely available for redistribution 